\title{
Infección por dengue: una causa frecuente de síndrome febril en pacientes de Quibdó, Chocó, Colombia
}

\author{
Berta Nelly Restrepo ${ }^{1}$, Leidy Diana Piedrahita', Ivony Yireth Agudelo', \\ Katherine Marín'², Ruth Ramírez
}

\footnotetext{
1 Instituto Colombiano de Medicina Tropical, Universidad CES, Sabaneta, Colombia

2 Institución Universitaria Colegio Mayor de Antioquia, Medellín, Colombia

Institución en la que se llevó a cabo el trabajo:

Instituto Colombiano de Medicina Tropical, Universidad CES, Sabaneta, Colombia
}

Introducción. El municipio de Quibdó (Chocó) presenta condiciones sociales, climáticas y de servicios públicos que son propicias para la transmisión del dengue, sin embargo, son escasos los estudios sobre el problema en este lugar.

Objetivo. Determinar la frecuencia de dengue en pacientes con enfermedad febril aguda y comparar las características clínicas de los pacientes con dengue y los pacientes con otras enfermedades febriles.

Materiales y métodos. Se realizó un estudio de corte transversal entre enero de 2008 y marzo de 2010. La población de estudio estuvo conformada por pacientes con síndrome febril de siete o menos días de evolución que consultaron en hospitales de la ciudad. El diagnóstico de dengue se hizo por detección en suero de anticuerpos IgM, antígeno NS1, reacción en cadena de la polimerasa con transcriptasa inversa y aislamiento viral. Además, se recolectó información clínica y de laboratorio de los pacientes.

Resultados. Se captaron 469 pacientes con síndrome febril, de los cuales el 98,3 \% eran afrodescendientes. El 28,4\% de ellos tenía dengue. Se identificaron los cuatro serotipos del virus con predominio del DENV-1. La edad de los pacientes osciló entre 0 y 76 años. El 70,7 \% de los casos se clasificó como dengue sin signos de alarma. Síntomas como náuseas, vómitos, dolor abdominal, tos y congestión nasal fueron significativamente más frecuentes en los pacientes con otra enfermedad febril. Conclusiones. La vigilancia activa del síndrome febril agudo permitió la detección de casos de dengue no diagnosticados; la frecuencia observada de esta infección sugiere que la zona de estudio es de riesgo elevado para dengue.

Palabras clave: dengue, signos y síntomas, grupo étnico, Colombia.

doi: http://dx.doi.org/10.7705/biomedica.v35i1.2345

Dengue infection: A common cause of febrile syndrome in patients from Quibdó, Chocó, Colombia

Introduction: Quibdó, a municipality in Chocó, has poor public services and weather and social conditions that favor dengue transmission. However, there are few studies about this problem in this district.

Objective: To determine the frequency of dengue infection in patients with acute febrile syndrome and to compare clinical features among dengue infected patients with other febrile diseases.

Materials and methods: A cross-sectional study was conducted from January, 2008, to March, 2010. The study population comprised patients with febrile syndrome of seven or fewer days of evolution, recruited from hospitals in the town. Dengue diagnosis was made in serum samples by detection of IgM antibodies, NS1 antigen, reverse transcription polymerase chain reaction, and virus isolation. Clinical and laboratory information from the patients was obtained.

Results: During the study period, 469 patients with acute febrile syndrome were recruited, of whom $98.3 \%$ were Afro-descendant. Dengue fever was found in $28.4 \%$ of the cases. Four dengue serotypes were identified with DENV-1 predominance. The ages ranged from zero to 76 years. From all patients, $70.7 \%$ of cases were classified as dengue without warning signs. Nausea, vomiting, abdominal pain, cough, nasal congestion and sore throat were significantly more frequent in patients with another febrile disease.

\section{Contribución de los autores:}

Berta Nelly Restrepo: formulación del proyecto de investigación, procesamiento de la información y elaboración del manuscrito Leidy Diana Piedrahita, Ivony Yireth Agudelo y Katherine Marín Velásquez: procesamiento de muestras, elaboración de las bases de datos y revisión del manuscrito

Ruth Ramírez: coordinación de la captación de casos, procesamiento de las muestras y elaboración de las bases de datos 
Conclusions: Active surveillance of the acute febrile syndrome allowed for the detection of undiagnosed cases of dengue; the observed frequency of this infection suggests that the study area has a high risk of dengue infection.

Key words: Dengue, signs and symptoms, ethnic group, Colombia.

doi: http://dx.doi.org/10.7705/biomedica.v35i1.2345

En Colombia, el dengue es un problema de salud pública que se ha incrementado de manera significativa en los últimos años, al pasar de un promedio anual de 11.387 casos en la década de 1980 a 1989 a 54.382 en los últimos cuatro años (2010 a 2013). Durante el 2010 se presentó la mayor epidemia del país con 151.774 casos: 5.420 de dengue grave y 170 con desenlace fatal (1). Más de 23 millones de personas están en riesgo de contraer el dengue, cuyo principal vector, Aedes aegypti, se ha detectado en el $77,6 \%$ de los municipios ubicados entre los 0 y los 2.200 msnm (2).

Chocó es uno de los cuatro departamentos que conforman la región de la Costa Pacífica colombiana. En este departamento el dengue es endemoepidémico con ciclos epidémicos cada 4 a 5 años (2) y un promedio anual de 58 casos durante el periodo de 2000 a 2009, lo que contrasta con el 2010, cuando se registraron 395 casos, de los cuales 15 fueron de dengue grave.

El municipio de Quibdó, capital del departamento, tiene condiciones propicias para la transmisión del dengue: una temperatura entre 26 y $30^{\circ} \mathrm{C}$, un elevado porcentaje de la población con necesidades básicas insatisfechas (89,8\%), bajas coberturas de acueducto y alcantarillado (35 y $15 \%$, respectivamente) (3) y elevados índices de infestación de Ae. aegypti (índice de Bretau de 27 \%) (Laboratorio Departamental de Salud Pública, Chocó. Consolidado de índices aédicos pre-intervención); sin embargo, la incidencia de dengue es baja comparada con la del resto de la Región Pacífica. Durante el 2010 se notificaron 2.813 casos de dengue en la región de la Costa Pacífica (1), es decir, una tasa de incidencia por 100.000 habitantes de 291,8 , y en el municipio de Quibdó se notificaron en ese mismo año 29 casos para una tasa de incidencia de 132,7 por 100.000 habitantes, es decir, casi la mitad. Estos datos permiten sospechar que el problema del dengue está subestimado en este municipio.

\footnotetext{
Correspondencia:

Berta Nelly Restrepo, Universidad CES, Carrera 43 A N 52 S-99, Sabaneta, Colombia

Teléfono: (574) 305 3500, extensión 2293; fax: (574) 3014258 brestrepo@ces.edu.co

Recibido: 27/03/14; aceptado: 03/10/14
}

En este contexto, el presente estudio se llevó a cabo en el municipio de Quibdó, con el fin de determinar la frecuencia de dengue en pacientes con enfermedad febril aguda y comparar las características clínicas de los pacientes con dengue y las de aquellos con otras enfermedades febriles. Estos datos pueden contribuir a mostrar la magnitud de la circulación del virus del dengue en Quibdó y a establecer la frecuencia de la enfermedad en pacientes con diagnóstico de síndrome de enfermedad febril, dada la alta prevalencia de muchas enfermedades transmisibles febriles en esta zona.

\section{Materiales y métodos}

\section{Área de estudio}

El presente estudio se llevó a cabo en las instituciones de salud ubicadas en la zona urbana del municipio de Quibdó, capital del departamento del Chocó, el cual está ubicado al noroccidente de Colombia en la región de la Costa Pacífica, entre los $04^{\circ} 00^{\prime} 50^{\prime \prime}$ y $08^{\circ} 41^{\prime} 32^{\prime \prime}$ de latitud norte y los $76^{\circ} 02^{\prime} 57^{\prime \prime}$ y $77^{\circ} 53^{\prime}$ ' $38^{\prime \prime}$ de longitud oeste, con una superficie de $46.530 \mathrm{~km}^{2}$ (3) (figura 1). La temperatura promedio es de $27^{\circ} \mathrm{C}$ y es la región con la mayor pluviosidad del planeta (3). La población

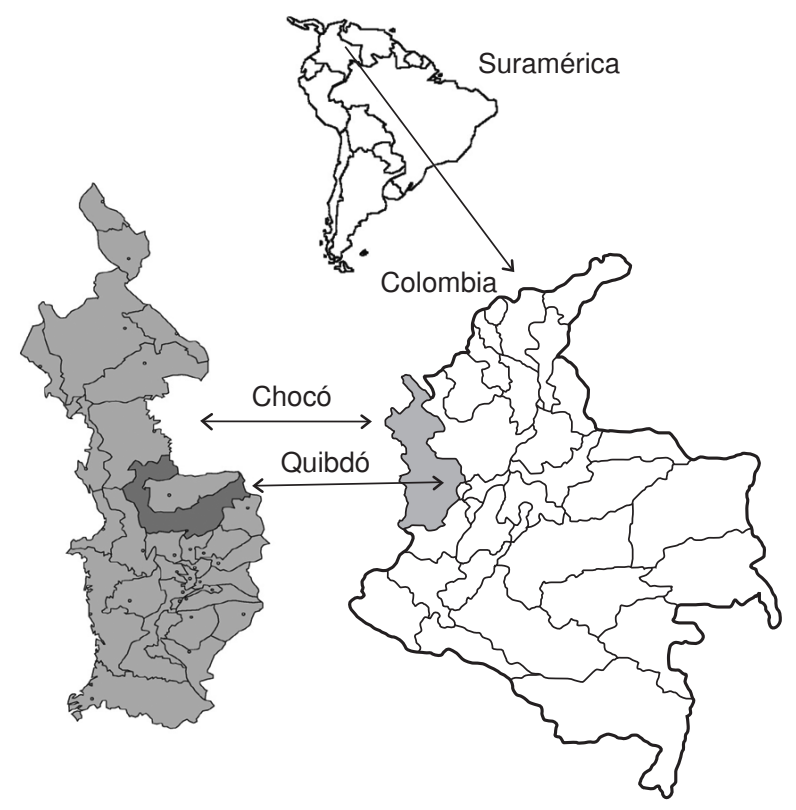

Figura 1. Ubicación del municipio de Quibdó, Chocó, Colombia 
del departamento es de 476.173 habitantes, en tanto que el municipio de Quibdó, ubicado a 43 msnm, está poblado por 112.886 habitantes (4), de los cuales el 95,3 \% son afrocolombianos, 2,3\%, mestizos y blancos y $1,4 \%$, indígenas (5).

\section{Tipo de estudio}

Se hizo un estudio descriptivo de corte transversal y prospectivo entre el 2008 y el 2010.

\section{Población de estudio}

La población de estudio estuvo constituida por pacientes que cumplían con el criterio de inclusión, es decir, con fiebre de 7 días o menos de evolución que demandaron atención médica en el periodo estudiado. Se excluyeron los pacientes con diagnóstico confirmado de paludismo o malaria.

Una auxiliar de enfermería visitaba en forma periódica las instituciones de salud para captar a los pacientes que cumplieran con el criterio de inclusión. Posteriormente, se les tomaba una muestra de $7 \mathrm{ml}$ de sangre periférica en la fase aguda de la enfermedad y otra muestra en la fase convaleciente (14 a 21 días después del inicio de los síntomas).

Las muestras se centrifugaron y los sueros se conservaron a $4{ }^{\circ} \mathrm{C}$ para enviarlos posteriormente al Instituto Colombiano de Medicina Tropical de la Universidad CES con el fin de procesarlos. Los datos demográficos de edad y sexo, así como la clasificación de la forma clínica del dengue, el tipo de infección, los signos y síntomas, los hallazgos de laboratorio y el conteo de plaquetas y hematocrito se obtuvieron de las historias clínicas de los pacientes y con ellos se diligenció un formulario elaborado para tal fin.

A los pacientes se les solicitó consentimiento informado por escrito para participar en el estudio y para menores de 18 años de edad este se solicitó a los padres o tutores. El estudio fue aprobado por el Comité de Bioética del Instituto Colombiano de Medicina Tropical.

\section{Diagnóstico del dengue}

Las muestras de suero recolectadas durante la fase aguda se procesaron para la detección del ARN del virus, el antígeno NS1, los anticuerpos IgM e lgG, y para el aislamiento viral. En la fase de convalecencia se hizo la detección de los anticuerpos lgM e lgG.

La detección del ARN del virus del dengue se determinó según el procedimiento descrito por Lanciotti, et al. (6), y modificado por Harris, et al. (7).
Para el aislamiento viral se utilizó una línea celular C6/36 de mosquitos Aedes albopictus según lo descrito por Gubler, et al. (8). El antígeno viral NS1 se obtuvo con el estuche SD Dengue Duo (Standard Diagnostics). La detección de los anticuerpos (Ac) IgM e IgG se hizo con los estuches DEN IgM capture ELISA (Panbio, Brisbane, Australia), DEN IgG capture ELISA (Panbio, Brisbane, Australia) y el SD Dengue Duo (Standard Diagnostics).

\section{Definición de caso}

El caso positivo de dengue se definió como un paciente febril en quien se hubiera detectado el virus por reacción en cadena de la polimerasa con transcriptasa inversa (reverse transcription polymerase chain reaction, RT-PCR), el aislamiento viral o el antígeno NS1, o la presencia de anticuerpos IgM en la muestra de la fase aguda, o seroconversión de anticuerpos IgM entre la fase aguda y la de convalecencia. Se consideró que había infección primaria cuando no se detectaban anticuerpos IgG en la fase aguda de la infección. Se consideró que había infección secundaria cuando se detectó la presencia de anticuerpos IgG en las muestras tomadas durante la fase aguda.

Los casos de dengue se clasificaron siguiendo los criterios de la Organización Mundial de la Salud (9). Se consideró que los pacientes presentaban otra enfermedad febril cuando los resultados de cualquiera de las pruebas utilizadas para el diagnóstico del dengue eran negativos.

\section{Análisis de los datos}

La base de datos se diligenció en Microsoft Exce ${ }^{\circledR}$ (Microsoft Corp., Redmond, WA) y su procesamiento se hizo mediante el programa Statistical Package for the Social Sciences, SPSS ${ }^{\circledR}$, versión 21 (SPSS, Inc.01., Chicago, IL). Se calcularon las frecuencias absoluta y relativa de las variables cualitativas y la mediana y el rango de las variables cuantitativas. La comparación de las variables cualitativas se hizo mediante la prueba de ji al cuadrado y la de las variables cuantitativas mediante la prueba $U$ de Mann-Whitney. Se definió un nivel de significancia del $5 \%$.

\section{Resultados}

De los 469 pacientes de la muestra con fiebre de 7 días o menos de evolución, el 51,6 \% (242/469) eran hombres; el 98,3\% (461/469) eran afrodescendientes y el resto, mestizos. La mitad de la población de estudio tenía 15,5 años (rango=0-80 años) y 16 pacientes eran menores de un año. 


\section{Frecuencia del dengue}

El 28,4 \% (133/469) de los pacientes fueron positivos para dengue. En las mujeres la frecuencia fue de $31,3 \%(71 / 227)$ y en los hombres de $25,6 \%$ (62/242, $p=0,174)$. En todos los grupos de edad (quinquenales), se presentaron casos de dengue con frecuencias alrededor del $30 \%$, siendo el de menor frecuencia el de 40 a 44 años, con $9,1 \%$, y el de mayor frecuencia el de 60 a 64 años, con $53,3 \%$. La mitad de los pacientes tenía 16 años (rango $=0-76$ ), cuatro eran menores de un año y todos eran afrodescendientes.

Durante los tres años de estudio la frecuencia anual de casos de dengue fue la siguiente: en el 2008 fue de $13,4 \%$ (19/142), en el 2009, de $28,7 \%$ (60/209) y en el 2010, de 45,8 \% (54/118).

El diagnóstico de dengue se hizo por detección de anticuerpos lgM y del antígeno NS1 en más del $90 \%$ de los casos. Diez pacientes fueron positivos por RT-PCR y en cinco se aisló el virus. Once pacientes fueron positivos en dos pruebas (cuadro 1).

Los serotipos identificados por RT-PCR fueron el DENV-1 en cinco pacientes, en tanto que los serotipos DENV-2, DENV-3 y DENV-4 se identificaron cada uno en un paciente. Por aislamiento viral se detectaron el DENV-3 en tres pacientes, y el DENV-1 en dos. En el 2008 y el 2009 los serotipos identificados fueron el DENV-1 y el DENV-3, años en que circularon los dos serotipos simultáneamente. En el 2010 se identificaron los cuatro serotipos.

El 70,7\% (94/133) de los casos fueron clasificados como dengue sin signos de alarma y el $29,3 \%$, como dengue con signos de alarma; no se observaron casos de dengue grave. De los 131 pacientes con dengue a quienes se les hizo detección de anticuerpos IgG en la muestra de la fase aguda, 36,6 \% tenían infección secundaria.

Cuadro 1. Diagnóstico de los casos de dengue según prueba de diagnóstico, Quibdó, Chocó, Colombia

\begin{tabular}{lrr}
\hline Tipo de prueba & n & \multicolumn{1}{c}{$\%$} \\
\hline Aislamiento viral & 3 & 2,3 \\
RT-PCR & 6 & 4,5 \\
Antígeno NS1 & 31 & 23,3 \\
IgM & 82 & 61,7 \\
IgM + antígeno NS1 & 5 & 3,8 \\
IgM + RT-PCR & 3 & 2,3 \\
IgM + aislamiento viral & 2 & 1,5 \\
RT-PCR + antígeno NS1 & 1 & 0,8 \\
Total & 133 & 100,0 \\
\hline
\end{tabular}

Comparación entre los pacientes con dengue y aquellos con otra enfermedad febril

No hubo diferencias entre los pacientes con dengue y aquellos con otras enfermedades febriles en cuanto a la edad (mediana: 16 años (0-76) Vs. 15,5 años $(0-80), p=0,723)$, ni en cuanto al sexo: la proporción de mujeres en los casos de dengue fue de 46,6 \% $y$ en los pacientes con otras enfermedades febriles fue de $53,6 \%(p=0,158)$.

Los signos y síntomas clínicos más frecuentemente observados en los casos de dengue fueron cefalea, escalofrío, mialgias y artralgias, náuseas y vómitos y en los pacientes con otra enfermedad febril fueron cefalea y escalofrío, seguido de náuseas, vómitos y dolor abdominal (cuadro 2).

Al comparar los dos grupos se observó mayor frecuencia de síntomas gastrointestinales, como vómitos y dolor abdominal, y de síntomas respiratorios, como tos, congestión nasal y dolor de garganta, en los pacientes con otra enfermedad febril. Estas diferencias fueron significativas (cuadro 2).

Las manifestaciones hemorrágicas observadas en los pacientes con dengue fueron gingivorragia y petequias en 5 y 3 casos, respectivamente, y gingivorragia, petequias y epistaxis en los pacientes con otras enfermedades febriles, y aunque fueron más frecuentes en este último grupo, las diferencias no fueron estadísticamente significativas (cuadro 2).

Se obtuvo información del recuento de plaquetas en 83 pacientes con dengue y en 132 con otras enfermedades febriles, de los cuales 17 (20,5\%) y $19(14,4 \%)$, respectivamente, presentaron trombocitopenia (recuento de plaquetas $\leq 100.000$ por $\mathrm{mm}^{3}$ ). Se obtuvo información del recuento de leucocitos en 19 pacientes con dengue y en 25 con otras enfermedades febriles, de los cuales nueve $(47,4 \%)$ y $10(40,0 \%)$, respectivamente, presentaron leucopenia. No hubo diferencias estadísticamente significativas entre los grupos para estas dos variables. No se observó hemoconcentración en ninguno de los 44 participantes que tenían el dato del hematocrito. Ningún paciente falleció.

\section{Discusión}

Este es el primer estudio que se realiza para determinar la frecuencia de dengue en pacientes con enfermedad febril aguda y comparar las características clínicas entre los pacientes con dengue y los pacientes con otras enfermedades febriles en el municipio de Quibdó. Los datos mostraron 
Cuadro 2. Comparación de signos y síntomas en pacientes con dengue y en aquellos con otra enfermedad febril, Quibdó, Chocó, Colombia, 2008-2010

\begin{tabular}{|c|c|c|c|c|c|}
\hline \multirow[t]{2}{*}{ Signos y síntomas } & \multicolumn{2}{|c|}{ Dengue $(n=133)$} & \multicolumn{2}{|c|}{ Otra enfermedad febril $(n=336)$} & \multirow[t]{2}{*}{$\mathbf{p}$} \\
\hline & $\mathbf{n}$ & $\%$ & $\mathbf{n}$ & $\%$ & \\
\hline \multicolumn{6}{|l|}{ Estado general } \\
\hline Cefalea $^{a}$ & 117 & 92,1 & 288 & 90,3 & 0,435 \\
\hline Escalofrío & 88 & 66,2 & 234 & 69,6 & 0,472 \\
\hline Mialgias o artralgias $^{a}$ & 55 & 43,3 & 124 & 38,9 & 0,388 \\
\hline Dolor retrooculara & 20 & 15,7 & 52 & 16,3 & 0,102 \\
\hline Astenia & 19 & 14,3 & 37 & 11,0 & 0,310 \\
\hline \multicolumn{6}{|l|}{ Gastrointestinal } \\
\hline Náuseas y vómitos & 54 & 40,6 & 185 & 55,1 & 0,006 \\
\hline Dolor abdominal & 32 & 24,2 & 100 & 75,8 & 0,030 \\
\hline Anorexia & 26 & 19,5 & 48 & 14,3 & 0,363 \\
\hline \multicolumn{6}{|l|}{ Respiratorio } \\
\hline Tos & 28 & 21,1 & 127 & 37,8 & 0,002 \\
\hline Congestión nasal o faríngea & 22 & 16,5 & 85 & 25,3 & 0,031 \\
\hline Dolor de garganta ${ }^{a}$ & 13 & 10,2 & 56 & 17,6 & 0,005 \\
\hline \multicolumn{6}{|l|}{ Hemorragias } \\
\hline Hemorragia gingival & 5 & 3,8 & 26 & 7,7 & 0,233 \\
\hline Petequias & 3 & 2,3 & 11 & 3,3 & 0,590 \\
\hline Epistaxis & 0 & 0,0 & 4 & 1,2 & 0,088 \\
\hline
\end{tabular}

${ }^{a}$ El denominador es 127 ( $\geq 2$ años de edad)

una frecuencia de dengue del $28,4 \%$ durante todo el período. Los resultados del presente estudio concuerdan con los obtenidos en pacientes con síndrome febril que acudieron a instituciones de salud en países como Nicaragua, donde la frecuencia de dengue según dos estudios consultados fue de $60 \%$ (10) y de $18 \%$ (11); otros estudios han revelado las siguientes frecuencias en diferentes países: en Puerto Rico, 10,9 \% (12); en Colombia, 47,7\% (13); en Brasil, $54 \%$ (14) y, globalmente en las Américas, $22 \%$ (15). En Vietnam la frecuencia de infección aguda de dengue en el 2002 fue de $33,6 \%$ (16) y de $19,7 \%$ (17), en tanto que en India fue de $19,9 \%$ (18) y en Tailandia de 51,5\% (19).

La frecuencia anual de dengue en el presente estudio fue superior a la reportada en el municipio de Quibdó: 13,4 \% Vs. 0,004 \% en el 2008; 28,7\% Vs. $0,011 \%$ en el 2009 y $45,8 \%$ Vs. $0,13 \%$ en el 2010 (Grupo Funcional de Enfermedades Transmitidas por Vectores, Dirección de Vigilancia y Análisis del Riesgo en Salud Pública del Chocó). Esta diferencia podría deberse a que mediante la vigilancia activa de síndromes febriles es posible captar pacientes con dengue no diagnosticados en los servicios de salud. Es frecuente que exista subdiagnóstico del dengue debido, en parte, a la elevada frecuencia de infecciones indiferenciadas o subclínicas (20-22), o porque los pacientes consultan en las etapas iniciales de la enfermedad, cuando los síntomas coinciden con los de otras infecciones y el diagnóstico es difícil
(23). La escasa disponibilidad de las pruebas de diagnóstico no permite confirmar los casos y, por ende, la magnitud real del problema no se conoce. En el presente estudio se confirmaron 92 casos mediante la detección de anticuerpos IgM, y al agregar otras pruebas diagnósticas se confirmaron otros 41 casos, además, se pudieron identificar los serotipos circulantes del virus del dengue.

Al comparar las frecuencias de dengue reportadas en este estudio se encontró que fue mayor en los años en que los índices de infestación de las viviendas y de los depósitos con $A$. aegypi fueron mayores: $19,88 \%$ y $11,03 \%$ en el 2009 y $34,7 \%$ y $16,8 \%$ en el 2010 , aunque los índices de Breteau fueron menores en el 2010 que en el 2009: 27,93\% Vs. 31,95\% (Laboratorio Departamental de Salud Pública, Chocó. Consolidado de índices aédicos pre-intervención). Como se sabe, los índices de infestación elevados y la transmisión del dengue no necesariamente están relacionados (24).

En este estudio el espectro clínico de la enfermedad fue leve en la mayoría de los casos. Una posible explicación es el origen étnico de los pacientes. La ascendencia africana se ha asociado a la protección contra las formas graves de la enfermedad (2527) y en la zona de estudio más del $90 \%$ de la población es afrocolombiana y todos los pacientes lo eran. Las infecciones secundarias se asocian a una mayor aparición de formas complicadas (2830). En este estudio se observó poca frecuencia 
de infección secundaria (36,6 \%), lo que también puede ser un factor que favorece la manifestación leve de la enfermedad.

La frecuencia de manifestaciones respiratorias y gastrointestinales en los pacientes del estudio fue significativamente mayor en los casos de otras enfermedades febriles. Con relación a la frecuencia de síntomas respiratorios, los resultados fueron semejantes a lo descrito por otros autores $(13,23,31)$; sin embargo, se sabe de un estudio en escolares que encontró mayor frecuencia de estos síntomas en los casos de dengue (32). Por el contrario, en este estudio la frecuencia de los síntomas gastrointestinales, como vómito y dolor abdominal, difiere de otros estudios que afirman que dichos síntomas son frecuentes en los casos de dengue $(33,34)$ y, además, hacen parte de los signos de alarma por su asociación con la gravedad de la infección por el virus del dengue (9). Esta discordancia podría explicarse parcialmente por la ocurrencia simultánea de dengue y otras infecciones respiratorias virales (12) o gastrointestinales, o porque en el caso de los síntomas respiratorios no se hace una búsqueda sistemática de estos, lo que puede repercutir en que su frecuencia en los casos de dengue esté poco documentada.

En conclusión, la vigilancia activa de los pacientes con síndromes febriles que acudieron a las instituciones de salud permitió la detección de casos de dengue no diagnosticados en los servicios de salud; la frecuencia observada de esta infección sugiere que la población de Quibdó tiene un riesgo elevado de dengue. Además, dicha vigilancia contribuyó al diagnóstico diferencial frente a otras enfermedades infecciosas de esta población. Estos datos pueden servir para orientar las medidas de prevención y control del dengue en la zona de estudio.

Con relación a la gravedad de la infección, aunque fue más común la forma leve, este hallazgo debe verse con precaución dada la información insuficiente sobre los resultados de laboratorio.

Por último, se recomienda continuar con este tipo de estudios, ampliando el diagnóstico a otros agentes etiológicos para conocer el perfil de los síndromes febriles en aras del manejo adecuado de los casos. También se sugiere llevar a cabo estudios de población que permitan determinar la magnitud e importancia del dengue en el municipio de Quibdó.

\section{Agradecimientos}

Al Laboratorio Departamental de Salud Pública de Chocó, a las directivas del Hospital Departamental
"San Francisco de Asís", al hospital local "Ismael Roldán Valencia", a Comfachocó, a Caprecom y a Coomeva, por permitir la captación de los casos.

\section{Conflicto de intereses}

Los autores declaramos que no existieron conflictos de intereses en la realización y difusión de este trabajo.

\section{Financiación}

Este trabajo fue financiado por el Instituto Colombiano de Medicina Tropical de la Universidad CES.

\section{Referencias}

1. Instituto Nacional de Salud. Estadística en Salud Pública. Vigilancia rutinaria. Fecha de consulta: 10 de enero de 2014. Disponible en: http://www.ins.gov.co/?idcategoria=82534\#.

2. Padilla JC, Rojas DP, Sáenz-Gómez R. Dengue en Colombia. Epidemiología de la reemergencia a la hiperendemia. 1aㅡ ed. Bogotá: Guías de Impresión Ltda.; 2012.

3. Gobernación del Chocó. Plan de Desarrollo 2012-2015. Departamento de Chocó. Un nuevo Chocó para vivir. Fecha de consulta: 5 de febrero de 2014. Disponible en: http:// educon.javeriana.edu.co/ofi/documentos/regionalizacion/ Planes\%20desarrollo/Departamentos/CHOCO\%20 2012_2015\%20PRELIMINAR.pdf.

4. Gobernación del Chocó. Fortalecimiento institucional. Fecha de consulta: 5 de febrero de 2014. Disponible en: http://www.choco.gov.co/apc-aa-files/65623435386535663 737303939383165/INTRODUCCION_DEFINITIVA.pdf.

5. Departamento Administrativo Nacional de Estadística. Censo general 2005. Perfil Quibdó, Chocó. Fecha de consulta: 5 de febrero de 2014. Disponible en: http:// www.dane.gov.co/files/censo2005/PERFIL_PDF_ CG2005/27001T7T000.PDF.

6. Lanciotti RS, Calisher CH, Gubler DJ, Chang GJ, Vorndam AV. Rapid detection and typing of dengue viruses from clinical samples by using reverse transcriptasepolymerase chain reaction. J Clin Microbiol. 1992;30:545-51.

7. Harris E, Roberts TG, Smith L, Selle J, Kramer LD, Valle $\mathrm{S}$, et al. Typing of dengue viruses in clinical specimens and mosquitoes by single-tube multiplex reverse transcriptase PCR. J Clin Microbiol. 1998;36:2634-9.

8. Gubler DJ, Kuno G, Sather GE, Vélez M, Oliver A. Mosquito cell cultures and specific monoclonal antibodies in surveillance for dengue viruses. Am J Trop Med Hyg. 1984;33:158-65.

9. World Health Organization. Dengue and dengue haemorrhagic fever. Geneva: WHO; 2008. Fecha de consulta: 12 de enero de 2014. Disponible en: http://www.who.int/ mediacentre/factsheets/fs117/en/.

10. Harris E, Videa E, Pérez L, Sandoval E, Téllez Y, Pérez ML, et al. Clinical, epidemiologic, and virologic features of dengue in the 1998 epidemic in Nicaragua. Am J Trop Med Hyg. 2000;63:5-11.

11. Balmaseda A, Saborio S, Téllez Y, Mercado JC, Pérez $\mathrm{L}$, Hammond $\mathrm{SN}$, et al. Evaluation of immunological markers in serum, filter-paper blood spots, and saliva for 
dengue diagnosis and epidemiological studies. J Clin Virol 2008;43:287-91. http://dx.doi.org/10.1016/j.jcv.2008.07.016

12. Lorenzi OD, Gregory CJ, Santiago LM, Acosta H, Galarza IE, Hunsperger $\mathbf{E}$, et al. Acute febrile illness surveillance in a tertiary hospital emergency department: Comparison of influenza and dengue virus infections. Am J Trop Med Hyg. 2013:88:472-80. http://dx.doi.org/10.4269/ajtmh.12-0373

13. Díaz FA, Martínez RA, Villar LA. Criterios clínicos para diagnosticar el dengue en los primeros días de enfermedad. Biomédica. 2006;26:22-30. http://dx.doi.org/10.7705/ biomedica.v26i1.1391

14. Cordeiro MT, Silva AM, Brito CA, Nascimento EJ, Magalhaes MC, Guimaraes GF, et al. Characterization of a dengue patient cohort in Recife, Brazil. Am J Trop Med Hyg. 2007;77:1128-34.

15. Daumas RP, Passos SR, Oliveira RV, Nogueira RM, Georg I, Marzochi KB, et al. Clinical and laboratory features that discriminate dengue from other febrile illnesses: A diagnostic accuracy study in Rio de Janeiro, Brazil. BMC Infect Dis. 2013;13:77. http://dx.doi.org/10.1186/1471-2334-13-77

16. Phuong $\mathbf{H L}$, de Vries $\mathbf{P J}$, Nga TT, Giao PT, Hung LQ, Binh TQ. Dengue as a cause of acute undifferentiated fever in Vietnam. BMC Infect Dis. 2006;6:123. http://dx.doi. org/10.1186/1471-2334-6-123

17. Thai KT, Phuong HL, Thanh Nga TT, Giao PT, Hung le $Q$, Van Nam N, et al. Clinical, epidemiological and virological features of Dengue virus infections in Vietnamese patients presenting to primary care facilities with acute undifferentiated fever. J Infect. 2010; 60:229-37. http://dx.doi.org/10. 1016/j.jinf.2010.01.003

18. Sharma Y, Kaur M, Singh S, Pant L, Kudesia M, Jain S. Seroprevalence and trend of dengue cases admitted to a government hospital, Delhi - 5-year study (2006-2010): A look into the age shift. Int J Prev Med. 2012;3:537-43.

19. Lertanekawattana S, Anantapreecha S, Jiraphongsa C, Duan-ngern P, Potjalongsin S, Wiittayabamrung W, et al. Prevalence and characteristics of dengue and chikungunya infections among acute febrile patients in Nong Khai Province, Thailand. Southeast Asian J Trop Med Public Health. 2013;44:780-90.

20. Burke DS, Nisalak A, Johnson DE, Scott RM. A prospective study of dengue infections in Bangkok. Am J Trop Med Hyg. 1988;38:172-80.

21. Balmaseda A, Hammond SN, Pérez L, Téllez Y, Saborío SI, Mercado JC, et al. Serotype-specific differences in clinical manifestations of dengue. Am J Trop Med Hyg. 2006;74:449-56

22. Capeding RZ, Brion JD, Caponpon MM, Gibbons RV, Jarman RG, Yoon IK, et al. The incidence, characteristics, and presentation of dengue virus infections during infancy. Am J Trop Med Hyg. 2010;82:330-6. http://dx.doi.org/10. 4269/ajtmh.2010.09-0542
23. Potts JA, Rothman AL. Clinical and laboratory features that distinguish dengue from other febrile illnesses in endemic populations. Trop Med Int Health. 2008;13:132840. http://dx.doi.org/10.1111/j.1365-3156.2008.02151.x

24. Sulaiman S, Pawanchee ZA, Arifin Z, Wahab A. Relationship between Breteau and House indices and cases of dengue/dengue hemorrhagic fever in Kuala Lumpur, Malaysia. J Am Mosq Control Assoc. 1996;12:494-6.

25. Bravo JR, Guzmán MG, Kourí G. Why dengue haemorrhagic fever in Cuba? Individual risk factors for dengue haemorrhagic fever/dengue shock syndrome (DHF/DSS). Trans R Soc Trop Med Hyg. 1987;81:816-20.

26. Blanton RE, Silva LK, Morato VG, Parrado AR, Dias JP, Melo PR, et al. Genetic ancestry and income are associated with dengue hemorrhagic fever in a highly admixed population. Eur J Hum Genet. 2008;16:762-5. http://dx.doi. org/10.1038/ejhg.2008.4

27. Restrepo BN, Ramírez R, Agudelo-Flórez P, Avendaño P, Chacón JC, Rojas W, et al. Características clínicas y niveles de citocinas en pacientes con dengue y su relación con la raza. Colombia. Rev Biomed. 2010;21:137-47.

28. Halstead SB. Pathogenesis of dengue: Challenges to molecular biology. Science. 1988;239:476-81. http://dx.doi. org/10.1126/science.3277268

29. Kliks SC, Nimmanitya S, Nisalak A, Burke DS. Evidence that maternal dengue antibodies are important in the development of dengue hemorrhagic fever in infants. Am J Trop Med Hyg. 1988;38:411-9.

30. Wahala WM, Silva AM. The human antibody response to dengue virus infection. Viruses. 2011;12:2374-95. http://dx. doi.org/10.3390/v3122374

31. Mustafa B, Hani AW, Chem YK, Mariam M, Khairul AH, Abdul Rasid K, et al. Epidemiological and clinical features of dengue versus other acute febrile illnesses amongst patients seen at government polyclinics. Med $\mathrm{J}$ Malaysia. 2010;65:291-6.

32. Restrepo BN, Piedrahita LD, Agudelo IY, ParraHenao G, Osorio JE. Frequency and clinical features of dengue infection in a schoolchildren cohort from Medellín, Colombia. J Trop Med. 2012;2012:120496. http://dx.doi. org/10.1155/2012/120496

33. Pires-Neto R da J, de Sá SL, Pinho SC, Pucci FH, Teófilo CR, Evangelista PD, et al. Dengue infection in children and adolescents: Clinical profile in a reference hospital in northeast Brazil. Rev Soc Bras Med Trop. 2013;46:765-68. http://dx.doi.org 10.1590/0037-8682-1716-2013

34. Durán A, Ochoa E, Alcocer S, Gómez M, Millano M, Martínez $\mathrm{O}$, et al. Frequency of gastrointestinal signs and symptoms of dengue. Analysis of a cohort of 1484 patients. Invest Clin. 2013;54:299-310. 